\title{
ANIMAL BREEDING
}

\section{OPPORTUNITIES}

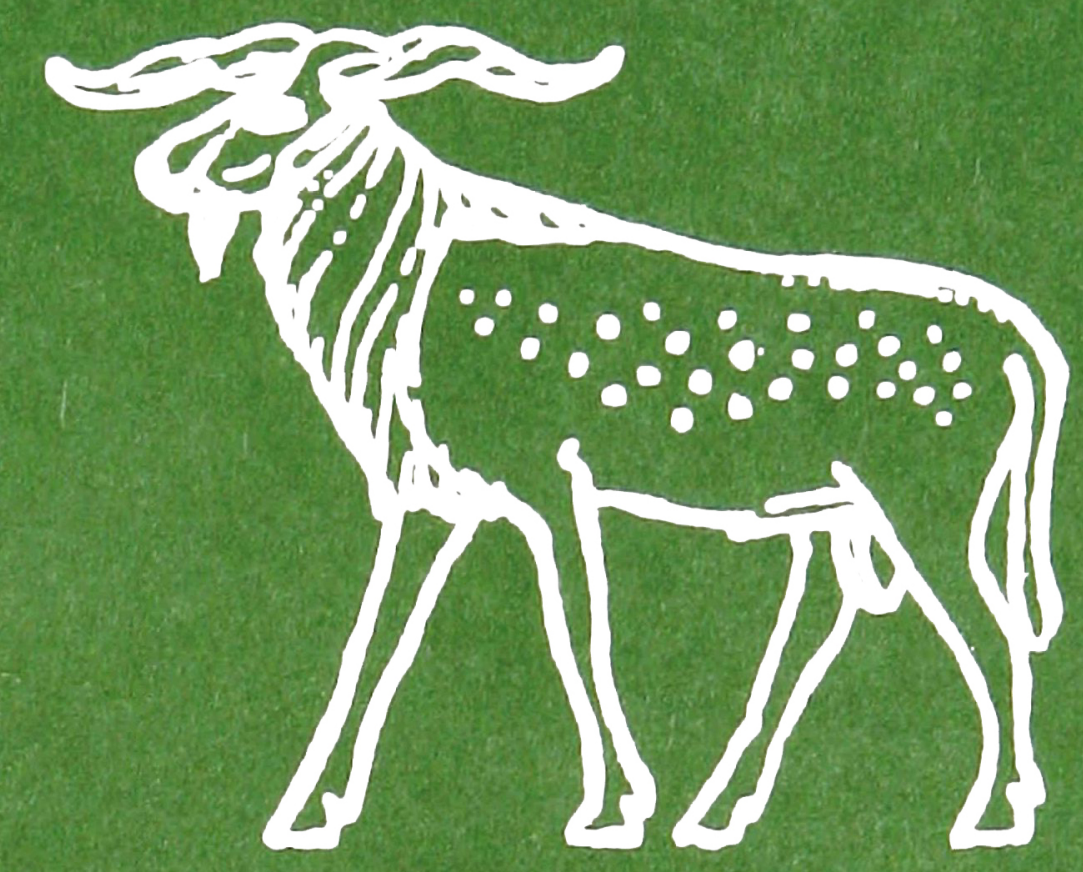

AN OCCASIONAL PUBLICATION

OF THE

BRITISH SOCIETY OF ANIMAL PRODUCTION

JOINTLY WITH

THE BRITISH POULTRY BREEDERS ROUNDTABLE

No. 12 
The British Society of Animal Production's Occasional Publication Series contains the full papers presented at certain symposia organized by the Society. A full list of titles is given on the back cover of this Publication. Copies may be obtained from the British Society of Animal Production, PO Box No. 3, Penicuik, Midlothian EH26 0RZ, Scotland. 


\title{
ANIMAL BREEDING
}

\section{OPPORTUNITIES}

Proceedings of a symposium organized jointly by THE BRITISH SOCIETY OF ANIMAL PRODUCTION and

THE BRITISH POULTRY BREEDERS ROUNDTABLE and held in Durham in September 1988

\section{BSAP OCCASIONAL PUBLICATION}

Number 12

\author{
BSAP \\ EDINBURGH \\ 1988
}


ISBN 0906562120

(C) 1988 THE BRITISH SOCIETY OF ANIMAL PRODUCTION

Printed in Great Britain by D. \& J. Croal Ltd., Haddington. 


\section{Pェ® Eace}

The sciences on which animal production is based are becoming increasingly communication amongst disciplines, advisers, and those in practice, becomes more and more difficult. This is particularly true of animal breeding. Its quantitative concepts often act as a deterrent to understanding by non-geneticists and as a result the opportunities animal breeding offers have not been fully realised. New statistical developments, which bring the potential of additional benefits, threaten to widen the gulf between animal breeding theory and practice and between animal breeding and the other animal sciences. Equally, whilst the recent advances in molecular genetics have re-emphasised the biological base of animal breeding, these too have often outpaced specialists in other disciplines, and are not widely understood.

This publication is a record of a meeting held to discuss present opportunities in animal breeding in a form intended to bridge the 'communication gulf'. The meeting dealt with the relevance of problems now being studied to the progress and future prospects for genetic improvement. It brought together specialists in a way that reflects the vigour of the science and the importance of animal breeding. The individual presentations were kept fairly general so as to provide background and understanding for non-specialists in any particular topic.

Acknowledgements

D. B. Marshall (Newbridge) Ltd.

Masterbreeders (Livestock Development) Ltd.

Meat and Livestock Commission

Milk Marketing Board

National Pig Development Co.

Pig Improvement Co.

Premier Breeders Ltd.

Ross Breeders Ltd.

The symposium was organized by the late Professor R. B. Land, Dr G Bulfield and Professor W. G. Hill.

Sessions were chaired by Dr G. Bulfield, Professor W. G. Hill and Dr G. Simm. 


\section{Molecular Blology and Genetic Manipulation}

Clark, A. J. Gene transfer in animal

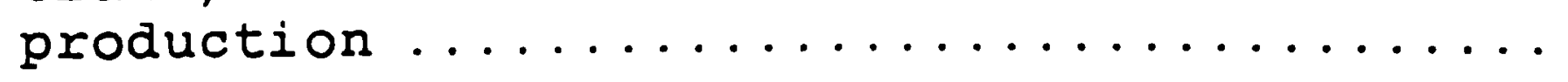

Brem, G. , Brenig, B. Müller, M. Kräusslich, H. and Winnacker, E-L. Production of transgenic pigs and possible application to pig breeding...

Salter, D. W. Gene insertion into the avian

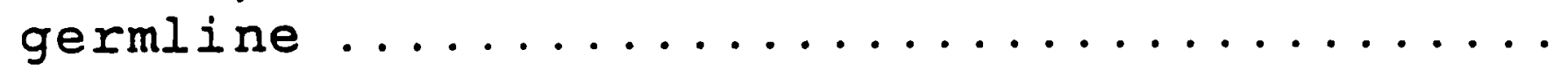

Maclean, N. and Penman, D. The application of gene technology to the improvement of fish

Smith, C. Integration of transgenes in

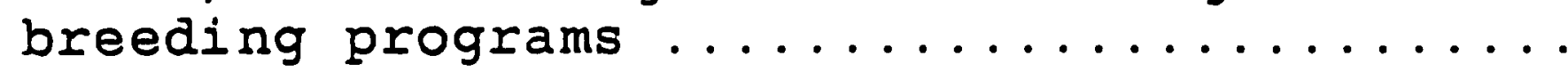

\section{Breeding Technology}

Hill, W. G. and Meyer, K. Developments in methods for breeding value and parameter

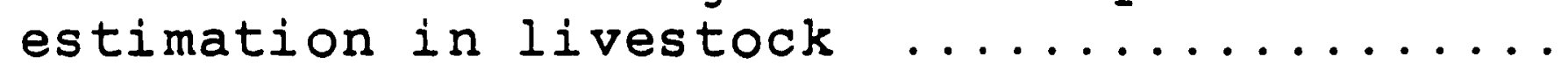

Pollak, E. J. New approaches to genetic

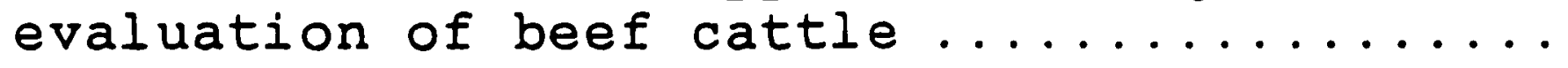

Webb, A. J. and Bampton, P. R. Impact of the new statistical technology on pig improvement

Freeman, A. E. Breeding programs in dairy cattle - current and future considerations

\section{Aspects of the Control of Growth}

Emmans, G. Genetic components of potential and

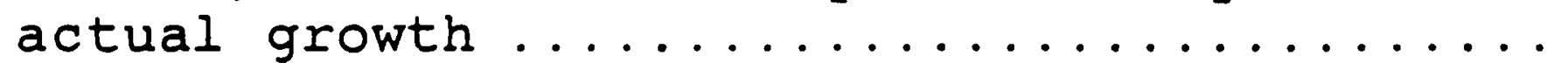

Goddard, C. Cellular growth - the key to

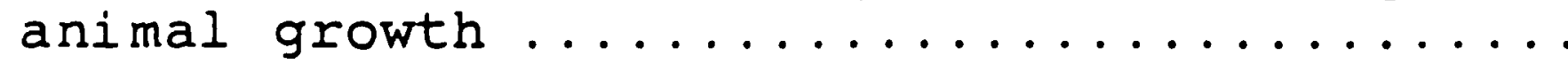

\section{Trait Improvement}

Flock, D. K. An assessment of progress with different stocks and methods of selection in

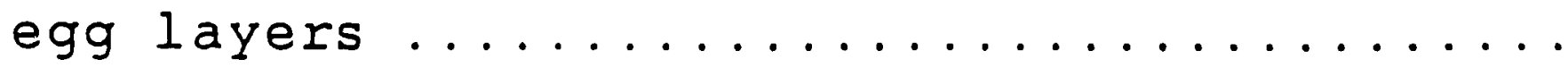

Simm, G. and Steane, D. E. Genetic improvement of meat production in sheep and cattle - current industry schemes and future

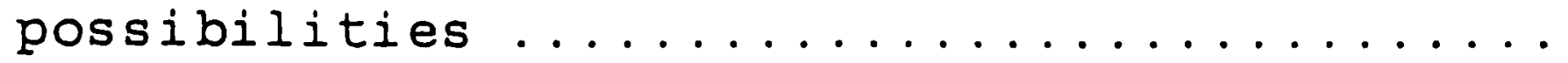

Johnson, R. K. and Neal, S. M. Opportunities and possible methods to improve reproduction in

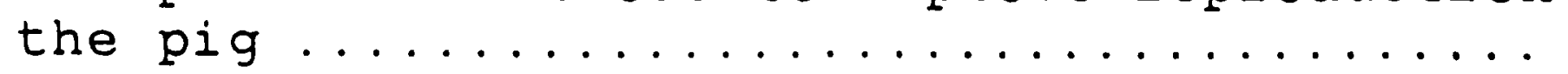

Siegel, P. B. and Dunnington, E. A. Long-term selection for meat production in poultry ... 
Trevelyan College, University of Durham

14 - 16 September, 1988

\section{E L E G A T E S}

Aarts, $\mathrm{Mr} \mathrm{H}$ Ainsworth, Ms $\mathrm{H}$ Albers, Dr G A A Al-Nakib, Dr F M S Asante, $\mathrm{Mr} \mathrm{E} \mathrm{A}$

Barton, $\mathrm{Mr} \mathrm{N} \mathrm{F}$ Baumbach, $\mathrm{Dr} W \mathrm{R}$ Baxter-Jones, Dr C Beattie, Mr S W Bell, Prof. F R Bhuiyan, $\operatorname{Mr} \mathrm{A} \mathrm{K} \mathrm{F} \mathrm{H}$ Bichard, Dr M Black, $\operatorname{Dr} W \mathrm{~J} M$ Borsting, $\mathrm{Mr} \mathrm{E}$ Bosch, Dr A Brenig, Dr Broadbent, Dr J S Broom, Miss $\mathrm{S}$ Bulfield, Dr G

Cameron, $\mathrm{Dr} \mathrm{N}$

Carr, Mr J

Challinor, $\mathrm{Mr} \mathrm{S} \mathrm{J}$

Christian, $\mathrm{Dr} \mathrm{L} \mathrm{L}$ Clark, Dr A J Clausen, $\mathrm{Mr} \mathrm{J}$ Coffey, Mr M P Collins-Lusweti Crump, Mr R E Curran, Dr M K

Darling, Mrs Joyce Davies, Mr D A R Dixon, $\mathrm{Mr} \mathrm{T}$ Donal, $\mathrm{Mr} \mathrm{R}$ Donkin, $\mathrm{Mr} E \mathrm{~F}$ Douaire, $\mathrm{Dr} M$ Drury, Mr D J

Ellis, $\mathrm{Mr} \mathrm{C} \mathrm{A}$ Emmans, $\mathrm{Mr} \mathrm{G}$ Evans, Dr P J Ewart, $\mathrm{Mr} J$
Cofok, The Netherlands MAFF (CSG)

Euribrid, Holland

APAD, ESCA

IAPG R, Roslin

Ross Breeders Ltd. American Cyanamid British United Turkeys Ltd. IAPG R-ERS University of Reading Wye College Pig Improvement Company Hon. Treasurer, BSAP Denmark IRTA - Catalonia, Spain Inst. of Animal Breeding, W. Germany ADAS, Great Westminster House Big Farm Weekly IAPG R, Roslin

IAPGR - ERS

University of Liverpool

ICI Chemicals \& Polymers Ltd.

Iowa State Univ./Swedish Univ. of Ag. Sciences IAPG R, Edinburgh

Danish Fur Breeders Association

Holstein Friesian Society

University of Zimbabwe

University of Edinburgh

University of London

BSAP

University of Liverpool

Institut de Selection Animale, France Medunsa, South Africa

N RA, France

AF RC/IAPG R

Harper Adams Agricultural College

APAD, ESCA

Agribusiness

Ross Breeders Ltd. 
Fisher, Dr C

Flock, Prof. D K

Flock, Mrs

Francis, Mr J

Freeman, Dr A E

Gilbert, Dr H

Goddard, Dr C

Green, Mr M

Gunn, Mrs $R$ G

Gunn, $\operatorname{Dr} R \mathrm{G}$

Guo, $\mathrm{Mr} \mathrm{K}$

Guy, Mr D R

Haley, $\operatorname{Dr} C$

Hall, Dr J

Hart, Dr I

Hartley, Mr G W

Hedemand, $\mathrm{Mr} \mathrm{J}$ E

Hill, Prof. W G

Hocking, $\operatorname{Dr} \mathrm{P} M$

Hogg, Mrs Sheena

Horn, Dr J P

Hoste, $\mathrm{Mr} \mathrm{S}$

Houdebine, Dr L M

Johnson, Miss C A

Johnson, $\operatorname{Dr} \mathrm{R} K$

Juga, Mr J

Kangasniemi, $\mathrm{Mr} \mathrm{R}$ Kanyia, Mr S

King, Prof. J W B

Knott, Miss S A

Leenstra, $\operatorname{Dr} F$

Liljedahl, Prof. L

Logan, Dr J

MacLeod, Ms I

Manson, Dr J M

Maragliano, $\mathrm{Dr} \mathrm{L}$

Martin, Mr D M

McAdam, Mr J

McCaffrey, Miss C

McCarthy, Prof. J

MeEvoy, $M r \mathrm{~T} G$

McGuirk, Dr

McKay, Dr J C

McLean, $\operatorname{Dr} \mathrm{N} A$

MeMahon, $\mathrm{Mr} \mathrm{R}$

MeManus, Ms C
IGAP, Pig \& Poultry Division

Lohmann Tierzucht GMBH, W. Germany

British United Turkeys Ltd.

Iowa State University

University of Newcastle-upon-Ttyne

IAPG R, Edinburgh

Farmers Weekly

Hon. Secretary, BSAP

IAPG R, Edinburgh

Meat \& Livestock Commission

IAPG R, Edinburgh

University of Newcastle-upon-Tyne

American Cyanamid Co.

D B Marshall (Newbridge)

National Institute of Animal Science, Denmark University of Edinburgh

IG AP, Roslin

BSAP

BOCM Silcock Ltd.

University of Reading

Institut National de Recherche Agronomique,

France

Wye College

University of Nebraska

Agricultural Research Centre, Finland

Agricultural Research Centre, Finland

Dept. of Genetics, University of Edinburgh

Consultant

IAPGR - ERS

Spelderholt (MAF, The Netherlands)

Swedish University of Agricultural Sciences

American Cyanamid

University of Edinburgh

Edinburgh School of Agriculture

National Pig Breeders Assoc., Italy

Cherry Valley Farms Ltd.

Ross Breeders Ltd.

Agricultural Institute, Belclare

University College Dublin

Agricultural Institute, Belclare

Premier Breeders

Ross Breeders Ltd.

University of Southampton

University of Edinburgh

University of Edinburgh 
Mercer, Dr J

Mews, Mr A

Mittal, Dr J P

More O'Ferrall, Dr G

Morris, Prof. T R

Morton, $\mathrm{Mr} \mathrm{C}$

Moruppa, $\mathrm{Mr}$ S M

Mueller, Dr

Murphy, Mr D

Nixey, $\mathrm{Mr} \mathrm{C}$

Ojala, Dr M

Onuora, $\mathrm{Mr} \mathrm{G}$

Orozco, Dr F

Payne, Dr W J A

Persaud, $\mathrm{Mr} \mathrm{P}$

Pirchner, Prof. F

Plastow, $\mathrm{Mr}$ G S

Pollak, Prof. J

Prescott, Prof. J H D

Protais, $\mathrm{Mr} \mathrm{M}$

Ricks, Dr C

Roberts, Dr C J

Roberts, $\mathrm{Dr} \mathrm{R} \mathrm{C}$

Roberts, Miss S

Roden, Miss J A

Rossouw, $\mathrm{Mr} \mathrm{P}$

Rowlett, Miss K A

Ruane, $\mathrm{Mr} \mathrm{J}$

Rymer, Mr J S

Salter, Dr D W

Salter, Mrs

Sellwood, Ms P

Sharp, Dr P J

Siegel, Prof. P B

Simm, Dr G

Simpson, Miss $\mathrm{P}$

Simpson, Dr S P

Smith, Prof C

Smith, Dr W K

Sorensen, $\mathrm{Dr} P$

Steane, $\mathrm{Mr} \mathrm{D} \mathrm{E}$

Stokholm, Mr K

Swanson, $\mathrm{Mr} \mathrm{G}$
National Pig Development Co.

RSPCA

Rowett Research Institute

Agricultural Institute, Grange

University of Reading

Ross Breeders Ltd.

University of Edinburgh

British Council, Hungary

Dept. of Agric. \& Food, Ireland

British United Turkeys Ltd.

Dept. of Animal Breeding, Finland

University of Reading

INIA, Spain

Edinburgh School of Agriculture

W. Germany

Dalgety plc

Cornell University, USA

President, BSAP

Institut de Selection Animale, France

American Cyanamid Co.

Home Office

Institute of Animal Genetics, Edinburgh

IAPG R, Edinburgh

University of Aberdeen

ADSRI, South Africa

University of Reading

IAPG R, Edinburgh

JSR Healthbred

U.S. Dept. of Agriculture, Michigan

Stock Magazine

AFRC, Physiology \& Genetics Research, Edinburgh

Virginia Polytechnic Inst. \& State University

Edinburgh School of Agriculture

Tropical Agriculture, Butterworths

IAPG R, Edinburgh

University of Guelph

West of Scotland College

National Inst. of Animal Science, Denmark

Meat \& Livestock Commission

Denmark

Milk Marketing Board 
Therkildsen, $\mathrm{Dr} \mathrm{N}$

Thompson, $\mathrm{Mr} \mathrm{R}$

Van Diepen, Mr J

Van Krey, Mr

Vincent, $\mathrm{Mr} \mathrm{J}$

Visscher, $\mathrm{Mr} \mathrm{P} M$

Webb, Dr A J

Wiener, $\mathrm{Dr} G$

Wolf, Dr B T

Woolliams, $\mathrm{Mr} \mathrm{J}$ A

Wray, Miss N

Young, Dr M

Young, Mrs R
Danish Fur Breeders Association IA PG R, Edinburgh

Christian Agric. College, The Netherlands Hypeco, The Netherlands

The Cobb Breeding Company

University of Edinburgh

Cotswold Pigs

Consultant.

Welsh Agricultural College

PG RS, Edinburgh

University of Edinburgh

APAD/ESCA 